\title{
SMAD4 mutations causing Myhre syndrome result in disorganization of extracellular matrix improved by losartan
}

\author{
Pasquale Piccolo ${ }^{1}$, Pratibha Mithbaokar ${ }^{1}$, Valeria Sabatino ${ }^{1}$, John Tolmie $^{2}$, Daniela Melis ${ }^{3}$, \\ Maria Cristina Schiaffino ${ }^{4}$, Mirella Filocamo ${ }^{5}$, Generoso Andria ${ }^{3}$ and Nicola Brunetti-Pierri ${ }^{\star, 1,3}$
}

Myhre syndrome (MS, MIM 139210) is a connective tissue disorder that presents with short stature, short hands and feet, facial dysmorphic features, muscle hypertrophy, thickened skin, and deafness. Recurrent missense mutations in SMAD4 encoding for a transducer mediating transforming growth factor $\beta$ (TGF- $\beta$ ) signaling are responsible for MS. We found that MS fibroblasts showed increased SMAD4 protein levels, impaired matrix deposition, and altered expression of genes encoding matrix metalloproteinases and related inhibitors. Increased TGF- $\beta$ signaling and progression of aortic root dilation in Marfan syndrome can be prevented by the antihypertensive drug losartan, a TGF- $\beta$ antagonists and angiotensin-II type 1 receptor blocker. Herein, we showed that losartan normalizes metalloproteinase and related inhibitor transcript levels and corrects the extracellular matrix deposition defect in fibroblasts from MS patients. The results of this study may pave the way toward therapeutic applications of losartan in MS.

European Journal of Human Genetics (2014) 22, 988-994; doi:10.1038/ejhg.2013.283; published online 8 January 2014

Keywords: myhre syndrome; SMAD4; losartan

\section{INTRODUCTION}

Myhre syndrome (MS, MIM 139210) is an autosomal dominant disorder presenting with short stature, laryngotracheal stenosis, brachydactyly, generalized muscle hypertrophy, joint stiffness, thick skin, and a distinctive facies. ${ }^{1}$ Cognitive disability, mixed conductive/ sensory hearing loss, and cardiac involvement are frequently reported. ${ }^{2,3}$ MS belongs to the group of acromelic dysplasias including Weill-Marchesani syndrome (WMS; MIM 277600, 608328, and 614819) and geleophysic dysplasia (GD; MIM 231050 and 614185). Molecular bases of these conditions have been recently elucidated and alterations in transforming growth factor $\beta$ (TGF- $\beta$ ) signaling have been recognized as a common pathogenic mechanism. ${ }^{4}$ Stiff skin syndrome (SSS, MIM 184900), which shares with MS increased skin thickness, joint stiffness, and short stature, is due to FBN1 mutations that result in increased TGF- $\beta$ signaling. ${ }^{5}$

Mutations affecting the codon for Ile500 of SMAD4 gene have been found in patients with MS and with the related laryngotracheal stenosis, arthropathy, prognathism, and short stature (LAPS) syndrome. ${ }^{3,6-8}$ The Ile500 residue is located in the Mad Homology 2 (MH2) domain of SMAD4 that is required for SMAD oligomerization and TGF- $\beta$ /Bone Morphogenetic Protein (BMP) signal transduction. ${ }^{9}$ Mutations of this residue result in perturbation of expression of both TGF- $\beta$ and BMP target genes that are involved in extracellular matrix (ECM) homeostasis. ${ }^{3,6}$ SMAD4 is a tumor suppressor gene involved in the development of various malignancies. ${ }^{10}$ Moreover, a large number of constitutional
SMAD4 loss of function mutations affecting all protein domains including $\mathrm{MH} 2^{11}$ are responsible for juvenile polyposis syndrome (JPS, MIM 174900) that may be combined with hereditary hemorrhagic telangiectasia (JPS-HTT, MIM 175050). ${ }^{11}$ In a previously reported family with SMAD4 mutations, aortic disease including aortic dilation and cystic medial necrosis have been described in addition to JPS, thus suggesting that SMAD4 haploinsufficiency may be responsible for the aortopathy. ${ }^{12}$ In contrast, SMAD4 mutations in MS appear to result in a gain of function, as shown by decreased ubiquitination and increased protein levels. $^{6}$

In the present study, we investigated the functional consequences of SMAD4 mutations on ECM and evaluated the efficacy of TGF- $\beta$ signaling antagonist losartan for correction of the ECM deposition defect.

\section{MATERIALS AND METHODS}

Patients MS1, MS3, and MS4

Clinical and molecular findings of these patients were reported elsewhere. ${ }^{2,3,13}$ Patients MS1 and MS3 were previously found to harbor the recurrent c.1498A $>$ G (p.Ile500Val) mutation, ${ }^{3}$ whereas patient $\mathrm{MS}^{2}$ carried the c.1486C > T (p.Arg496Cys) mutation in SMAD4 gene.

\section{Patient MS2}

Patient MS2 was followed from the age of 12 years and 7 months up to 30 years. This patient presented with short stature, 'happy face' appearance,

${ }^{1}$ Telethon Institute of Genetics and Medicine, Naples, Italy; ${ }^{2}$ Ferguson-Smith Department of Clinical Genetics, Yorkhill Hospital, Glasgow, UK; ${ }^{3}$ Department of Translational Medicine, Federico II University of Naples, Naples, Italy; ${ }^{4}$ Clinica Pediatrica, Istituto G. Gaslini, Genova, Italy; ${ }^{5}$ Centro di Diagnostica Genetica e Biochimica delle Malattie Metaboliche, Istituto G. Gaslini, Genova, Italy

${ }^{*}$ Correspondence: Dr N Brunetti-Pierri, Department of Translational Medicine, Federico II University of Naples and Telethon Institute of Genetics and Medicine, Via P. Castellino, 111, Napoli 80131, Italy. Tel: +39 081 6132361; Fax: +39 081 5609877; E-mail: brunetti@tigem.it

Received 27 March 2013; revised 4 November 2013; accepted 6 November 2013; published online 8 January 2014 
hypertelorism, epicanthal folds, aortic valve stenosis, severe bilateral sensorineural hearing loss, and speech delay. Skeletal findings included short limbs, small hands and feet, and joint contractures. She also had laryngotracheal stenosis and chronic respiratory infections. She had astigmatism and abnormalities of the fundus including blurred margins of the papilla, vascular congestion, and the arterovenous crossing sign. On the basis of clinical presentation, she was first diagnosed as GD but ADAMTSL2 and FBN1 sequencing revealed no mutations. Upon SMAD4 sequencing, she was found to harbor the recurrent c.1498A $>\mathrm{G}$ (p.Ile500Val) mutation.

The described mutations are based on the reference SMAD4 (NM_005359.5) accession. Newly described c.1486C > T (p.Arg496Cys) mutation has been submitted to dbSNP database (http://www.ncbi.nlm.nih. gov/SNP)

\section{Cell cultures and treatments}

Skin biopsies were obtained from all four MS patients. Control skin fibroblasts from Marfan syndrome (MFS) harboring FBN1 mutations were obtained from Coriell Institute for Medical Research (Camden, NJ, USA). Control wild-type fibroblasts were obtained from healthy subjects. Fibroblasts were cultured according to standard procedures and maintained in DMEM medium (EuroClone, Pero, Italy) with 10\% FBS and penicillin/streptomycin, in a humidified atmosphere containing $5 \% \mathrm{CO}_{2}$ at $37^{\circ} \mathrm{C}$. Losartan potassium salt (Sigma-Aldrich, St Louis, MO, USA) was dissolved into DMSO (SigmaAldrich) and used at the concentration of $200 \mu \mathrm{M}$ for 14 days. Treatments were performed by daily changes with supplemented maintenance medium. Recombinant human TGF- $\beta 1$ (Sigma-Aldrich) was reconstituted in $4 \mathrm{mM}$ $\mathrm{HCl}, 0.1 \%$ human serum albumin, and used at $10 \mathrm{ng} / \mathrm{ml}$ for $1 \mathrm{~h}$

\section{Western blotting}

Proteins from primary cultured fibroblasts were extracted in RIPA buffer according to standard procedures. Primary antibodies were: anti-SMAD4, antipSMAD2, anti-SMAD2 (Cell Signaling Technology, Danvers, MA, USA), and anti-Calnexin (Enzo Life Sciences, Farmingdale, NY, USA); secondary antibody was ECL anti-rabbit HRP (GE Healthcare, Waukesha, WI, USA). Analysis of band intensities was performed using Quantity One basic software (Bio-Rad Laboratories, Hercules, CA, USA).

\section{Real-time PCR}

Total RNA was extracted from primary cultured fibroblasts using RNeasy kit (Qiagen, Hilden, Germany) according to the manufacturer's instructions. RNA was reverse transcribed using a first-strand complementary deoxyribonucleic acid kit with random primers according to manufacturer's protocol (Life Technologies, Grand Island, NY, USA). The qPCR reactions were performed using Roche Light Cycler 480 system (Roche, Indianapolis, IN, USA). PCR reactions were performed with SYBR Green Master Mix (Roche). PCR conditions were as follows: preheating, $5 \mathrm{~min}$ at $95^{\circ} \mathrm{C} ; 40$ cycles of $15 \mathrm{~s}$ at $95^{\circ} \mathrm{C}, 15 \mathrm{~s}$ at $60^{\circ} \mathrm{C}$, and $25 \mathrm{~s}$ at $72^{\circ} \mathrm{C}$. Quantification results were expressed in terms of cycle threshold $\left(C_{\mathrm{t}}\right)$. The $C_{\mathrm{t}}$ values were averaged for each technical duplicate. For the expression analysis HPRT1, GAPDH, and B2M housekeeping genes were used as endogenous controls (reference markers) using LightCycler 480 software version 1.5. Differences between mean $C_{\mathrm{t}}$ values of tested genes and those of the reference gene were calculated as $\Delta C_{\mathrm{t}}$ gene $=C_{\mathrm{t}}$ gene $-C_{\mathrm{t}}$ reference. WT1 sample was used as calibrator and relative fold increase in expression levels was determined as $\mathrm{E}^{-\Delta \Delta \mathrm{Ct}}$, $\mathrm{E}$ being primer efficiency. The analyzed genes and relative primers were: $M M P 2$ for $5^{\prime}$-ATAACCTGGATGCC GTCGT-3'; MMP2 rev 5'-AGGCACCCTTGAAGAAGTAGC-3'; MMP9 for 5'-GAACCAATCTCACCGACAGG-3'; MMP9 rev 5'-GCCACCCGAGTGTAAC CATA-3'; MMP14 for 5'-GCAGAAGTTTTACGGCTTGCA-3'; MMP14 rev 5'TCGAACATTGGCCTTGATCTC-3'; SERPINE 1 for $5^{\prime}$-CCCTTTGCAGGATGGAACTA-3'; SERPINE1 rev 5'-TGGCAGGCAGTACAAGAGTG-3'; TIMP1 for 5'- GTCCCTGCGGTCCCAGATA- ${ }^{\prime}$; TIMP1 rev 5'-GTGGGAACAGGGTGG ACACT-3'; TIMP2 for 5'-CGACATTTATGGCAACCCTATCA-3'; TIMP2 rev 5'-GGGCCGTGTAGATAAACTCTATATCC-3'; TIMP3 for $5^{\prime}$-ATCACCTG GGTTGTAACTGCAA-3'; TIMP3 rev 5'-CGCTCCAGAGACACTCGTTCTT$3^{\prime}$; RECK for $5^{\prime}$-TGCAAGCAGGCATCTTCAAA-3'; RECK rev 5'-ACCGAGCC CATTTCATTTCTG- $3^{\prime}$. Each experiment was performed in triplicate.

\section{Immunofluorescence}

Primary skin fibroblasts were cultured for 14 days, fixed in 4\% PFA, incubated with blocking solution (1\% BSA, $50 \mathrm{mM} \mathrm{NH}_{4} \mathrm{Cl}$, PBS $\mathrm{pH}$ 7.4) for $1 \mathrm{~h}$ at room temperature without permeabilization. Anti-fibrillin 1 and anti-collagen type I (Millipore, Millerica, MA, USA) were used as primary antibody, and antimouse AlexaFluor-594 and anti-goat AlexaFluor-488 (Life Technologies, Carlsbad, CA, USA) as secondary antibodies, respectively. Nuclei were counterstained with DAPI (Life Technologies). Confocal images were obtained using LSM 710 confocal laser scanning microscope and ZEN 2008 software (Carl Zeiss, Oberkochen, Germany). Each experiment was performed at least in duplicate and at least five images per experiment were analyzed for each staining. Images from untreated samples and quantification of red intensity were performed by Image software (NIH, Bethesda, MD, USA). Ratio of red fluorescence intensity and red signal positive area was calculated. Sobel operator was used to calculate the threshold value for image segmentation with Matlab. Images from DMSO- and losartan-treated samples were analyzed using Leica MM Angiogenesis package (Leica Microsystems, Wetzlar, Germany) of MetaMorph software (MDS Analytical Technologies, Sunnyvale, CA, USA). MetaMorph software was used to evaluate: (i) microfibril length and (ii) area calculated in microns and square microns; (iii) segments, which were defined as the segments between fiber branching points and/or ends; (iv) branching points, which are the junction connecting segments; (v) nodes, which are connected blobs with thickness exceeding maximum width and are excluded from fiber length or area calculation.

\section{Statistical analyses}

Statistical significance was computed using the Student's two tail test or one sample two tail test. A $P$-value $<0.05$ was considered statistically significant. To test for lack of statistical significance, we adopted a pooled-variance two-sample $t$-statistic from a Bayesian formulation of the two-sided point null testing problem. ${ }^{14}$ We tested the hypothesis $\mathrm{H}_{0}: \mu_{1}=\mu_{2}$ against the alternative $\mathrm{H}_{1}: \mu_{1} \neq \mu_{2}$. Bayesian Factor (BF) was calculated as: $\mathrm{P}\left(\right.$ data| $\left.\mid \mathrm{H}_{0}\right) / \mathrm{P}\left(\right.$ data $\left.\mid \mathrm{H}_{1}\right)$. A $\mathrm{BF}>1$ provided evidence for $\mathrm{H}_{0}$.

\section{RESULTS}

SMAD4 mutations result in increased SMAD4 and phosphorylated SMAD2 proteins and impaired microfibril deposition

MS fibroblasts harboring the c.1498 A $>$ G (p.Ile500Val) (MS1, MS2, and MS3), and although at a lesser extent the c.1486C $>$ T (p.Arg496Cys) (MS4) mutation, exhibited increased SMAD4 protein levels compared with wild-type (WT) controls (Figure 1) that only showed detectable SMAD4 protein following TGF- $\beta$ stimulation (Supplementary Figure 1). Consistent with previous studies, 3,16 using primary cell lines including fibroblasts, SMAD4 western blotting resulted in detection of two bands, likely depending upon its post-translational modifications such as phosphorylation, ${ }^{17}$ acetylation, ${ }^{18}$ and ubiquitination. ${ }^{19}$ The increase in SMAD4 protein in MS cells is consistent with a previous study that showed elevated SMAD4 protein levels due to impaired ubiquitination in cells harboring the c.1499T $>C$ (p.Ile500Thr) mutation. ${ }^{6}$ MFS fibroblasts, used as positive control of the TGF- $\beta /$ SMAD pathway activation, also showed increased SMAD4 (Figure 1). MS fibroblasts showed increased phosphorylated SMAD2 (pSMAD2)/total SMAD2 ratio indicating constitutive activation of the TGF- $\beta /$ SMAD pathway (Figure 1) that is induced in WT controls only after TGF- $\beta$ stimulation (Supplementary Figure 1).

The binding of TGF- $\beta$ to its receptor results in recruitment and phosphorylation of SMAD2 that binds to SMAD4 to form a heterodimeric complex that migrates into the nucleus where it regulates the expression of a large set of genes encoding structural components of the ECM, growth factors, matrix metalloproteinases (MMPs), and their inhibitors. To investigate the consequences of SMAD4 mutations on ECM, we stained MS fibroblasts cultured 
for 14 days $^{20}$ for fibrillin 1 (FBN1) and collagen type I (COL1A1). Skin fibroblasts from MFS patients and from healthy subjects (WT) were used as controls. MS fibroblasts exhibited impaired microfibril deposition compared with WT controls (Figure 2a), as shown by a statistically significant $(t$-test: $P<0.01) 45 \%$ reduction of FBN1 staining (Figure 2b), whereas MFS fibroblasts showed a greater $60 \%$ reduction of fluorescence signal intensity compared with WT (Figure 2b). COL1A1 deposition was not impaired in either MS or MFS fibroblasts compared with WT (Figure 2a).
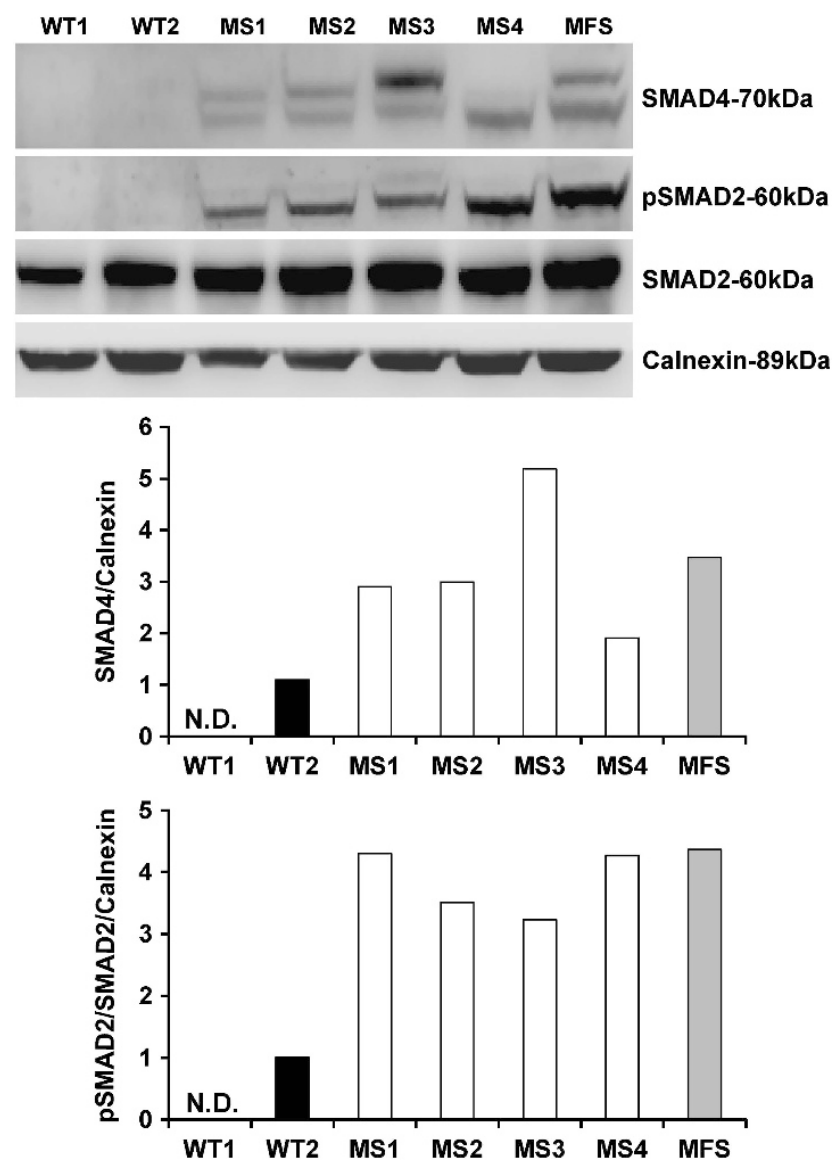

Figure 1 SMAD4 and phosphorylated SMAD2 protein levels in fibroblasts harboring SMAD4 mutations. Western blot analysis and band intensity quantification revealed increased SMAD4 and phosphorylated SMAD2 (pSMAD2)/SMAD2 ratio in MS fibroblasts (MS1, MS2, MS3, and MS4) compared with healthy control cells (WT1 and WT2) in which SMAD4 and pSMAD2 were barely detectable. Cells from Marfan syndrome patient (MFS) also showed increased SMAD4 and PSMAD2/SMAD2 ratio.

Figure 2 Fibroblasts harboring SMAD4 mutations have an ECM deposition defect. (a) WT, MS, and MFS fibroblasts were cultured for 14 days and stained for FBN1 (red) and COL1A1 (green) to evaluate ECM deposition. Nuclei were counterstained with DAPI (blue). Scale bar: $100 \mu \mathrm{m}$. (b) Quantification of fluorescence intensity showed impairment of microfibril deposition in MS fibroblasts compared with WT ( $t$-test: $\left.{ }^{* *} P<0.01\right)$. FBN1 deposition defect was more severe in MFS fibroblasts ( $t$-test: ${ }^{*} P<0.05$ compared with WT; ${ }^{\#} P<0.05$ compared with MS). Ratio between fluorescence intensity and fluorescence area is expressed as arbitrary units (AU) calibrated to WT.

Unbalanced expression of MMPs and related inhibitors in MS fibroblasts

The ratio between MMPs and their inhibitors, including tissue inhibitors (TIMPs), plasminogen activation inhibitor (PAI), and

a
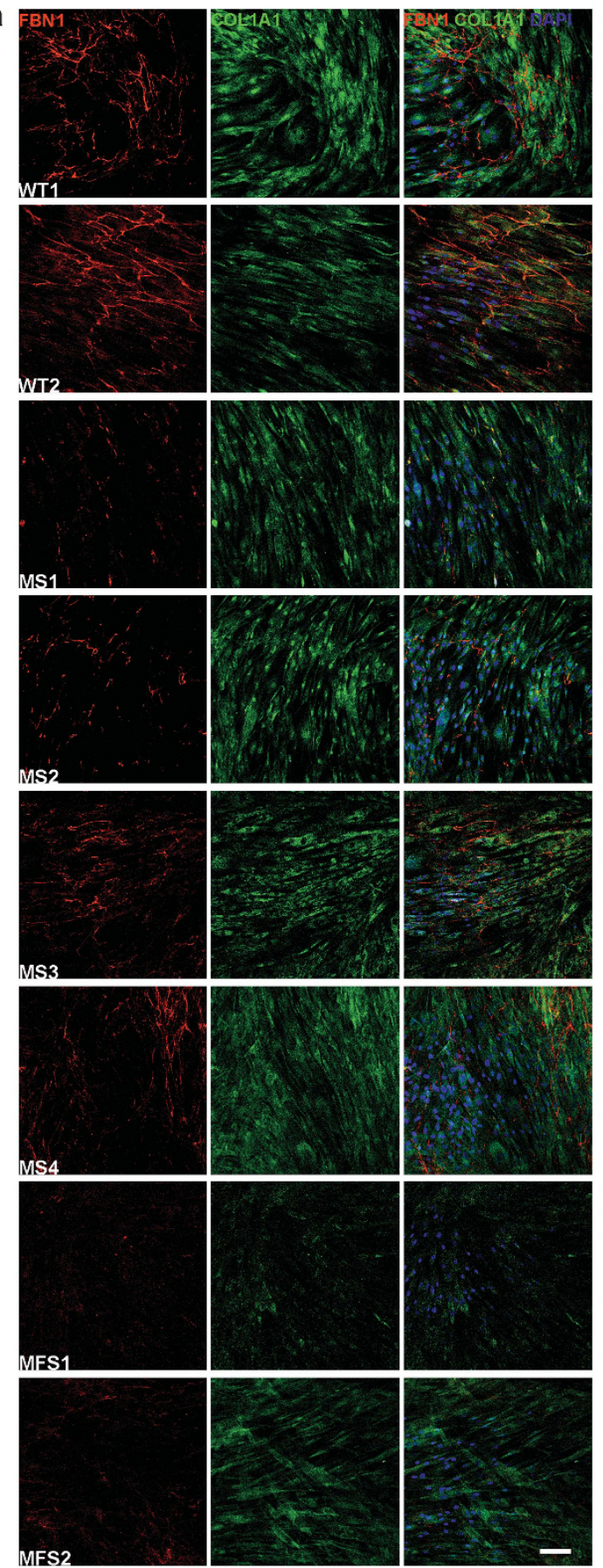

b

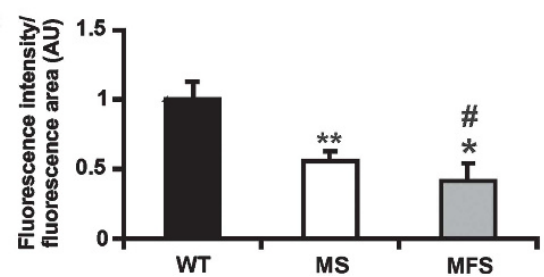



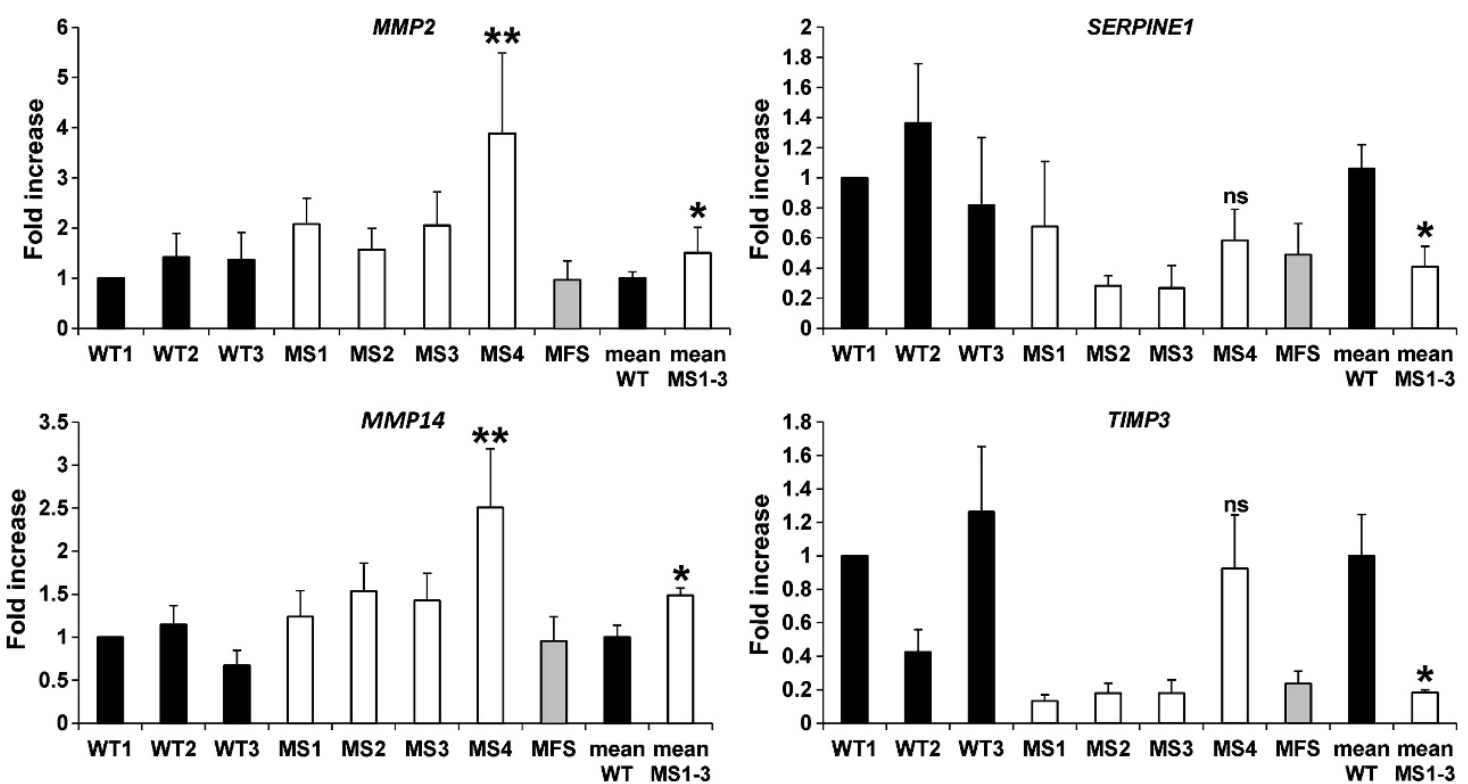

Figure 3 Altered expression of MMPs and relative inhibitors in MS fibroblasts. MS fibroblasts bearing the p.500lle>Val mutation (MS1, MS2, and MS3) showed increased expression of MMP2 and MMP14 and downregulation of SERPINE1 and TIMP3 compared with healthy controls (WT). MS4 showed significant increase in MMP2 and MMP14 expression, whereas SERPINE1 and TIMP3 transcripts were in the range of WT controls. MS1-3 represents an average of MS1, MS2, and MS3 cells. WT1 and WT mean samples were used as calibrator ( $t$-test: ${ }^{*} P<0.05$ vs WT; one sample $t$-test: ${ }^{*} P<0.01$ vs WT).
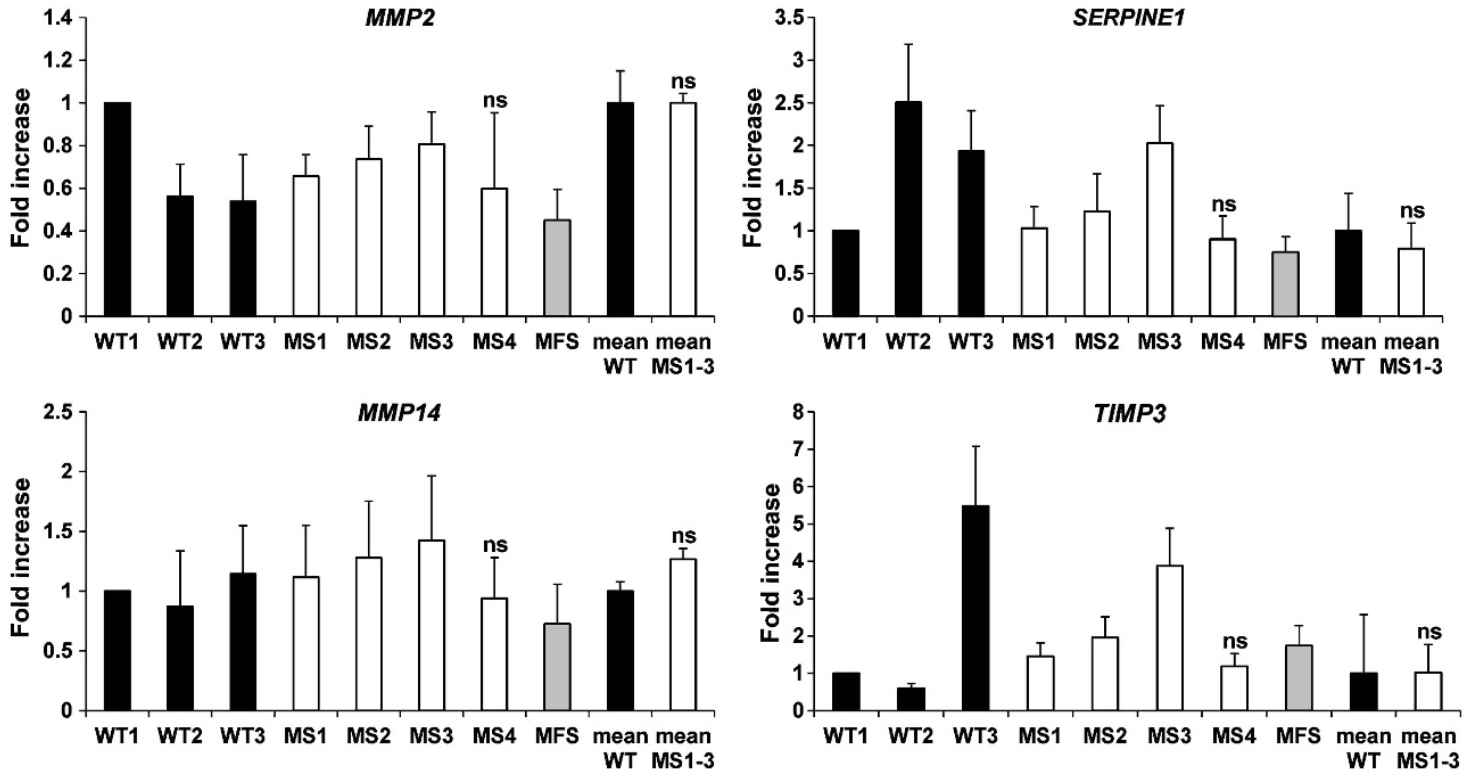

Figure 4 Losartan normalizes the imbalance between MMPs and related inhibitors in MS fibroblasts. Expression analysis of MMP2, MMP14, SERPINE1, and TIMP3 by real-time PCR on losartan-treated MS, MFS, and WT fibroblasts, showed no significant differences. MS1-3 represents the average of MS1, MS2, and MS3. WT1 and WT mean were used as calibrators.

membrane-bound inhibitor (Reversion-inducing-cysteine-rich protein with Kazal motifs-RECK) is a major determinant of ECM homeostasis and remodeling. ${ }^{21}$ Given the impaired microfibril deposition (Figure 2), we hypothesized MMP/inhibitor imbalance is involved in ECM defect observed in MS fibroblasts. To test this hypothesis, by real-time PCR $(n=3)$, we evaluated in MS, MFS, and primary skin fibroblasts from healthy controls the expression levels of FBN1 degrading MMPs (MMP2, MMP9, and MMP14) ${ }^{22}$ and tissue MMP inhibitors (TIMP1, TIMP2, TIMP3, PAI-1-encoding gene SERPINE1, and RECK) that are under the control of the TGF- $\beta / \mathrm{SMAD}$ pathway. ${ }^{23-25}$ MS fibroblasts bearing p.500Ile > Val mutation (MS1, MS2, and MS3) showed a statistically significant 1.5-fold upregulation of MMP2 and MMP14 expressions and downregulation of SERPINE1 (2.4-fold) and TIMP3 (5.5-fold) compared with WT ( $t$-test: $P<0.05$ ) (Figure 3 ). MS4 cells were analyzed separately because they harbor a different mutation affecting the Arg496 residue that is directly involved in SMAD4 transcriptional activation activity ${ }^{21}$ and they exhibited upregulation of MMP2 (3.9-fold) and MMP14 
+DMSO
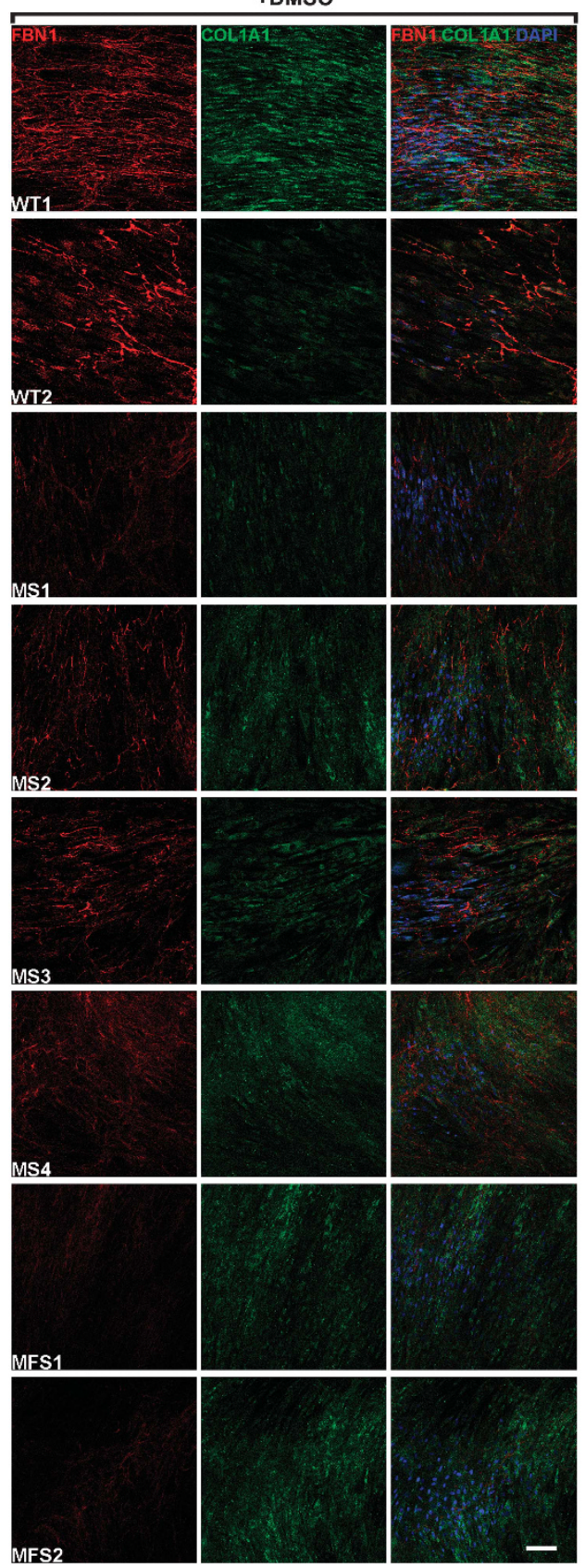

MFS2

Figure 5 Losartan improves microfibril deposition defect of MS fibroblasts. Fibroblasts from MS and MFS patients and from healthy controls (WT) were treated for 14 days with vehicle (DMSO) or $200 \mu \mathrm{M}$ losartan and then stained for FBN1 (red) and COL1A1 (green). Nuclei were counterstained with DAPI (blue). Losartan treatment improved microfibril deposition in both MS and MFS fibroblasts. Scale bar: $100 \mu \mathrm{m}$.

(2.5-fold) (one sample $t$-test: $P<0.01)$ compared with WT, but no significant differences in the expression of SERPINE1 and TIMP3 (Figure 3). MMP9 levels were below the limit of detection and no significant changes in expression between MS and control fibroblasts were detected in the other tested genes (data not shown).

\section{Losartan restores balance of MMPs and related inhibitors and} improves the ECM deposition defect

Losartan, an inhibitor of angiotensin-II type 1 receptor (AT1R), is used to treat aortic aneurysm and prevent dissection in MFS

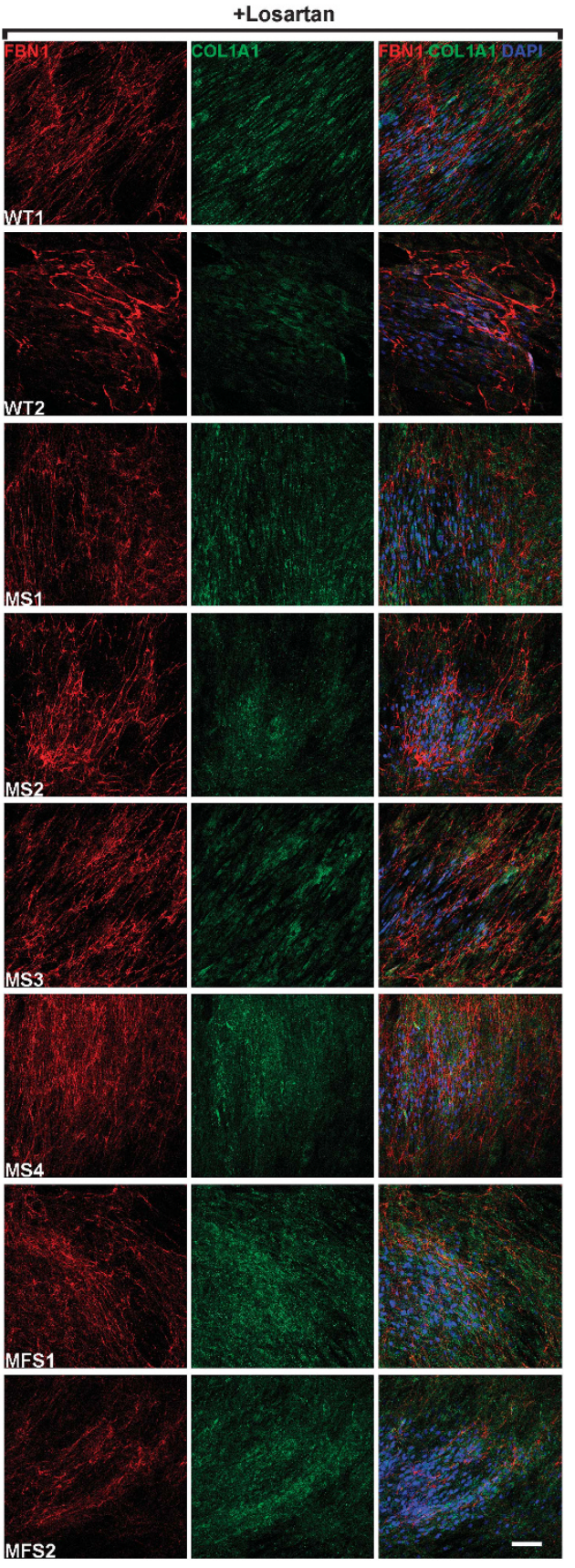



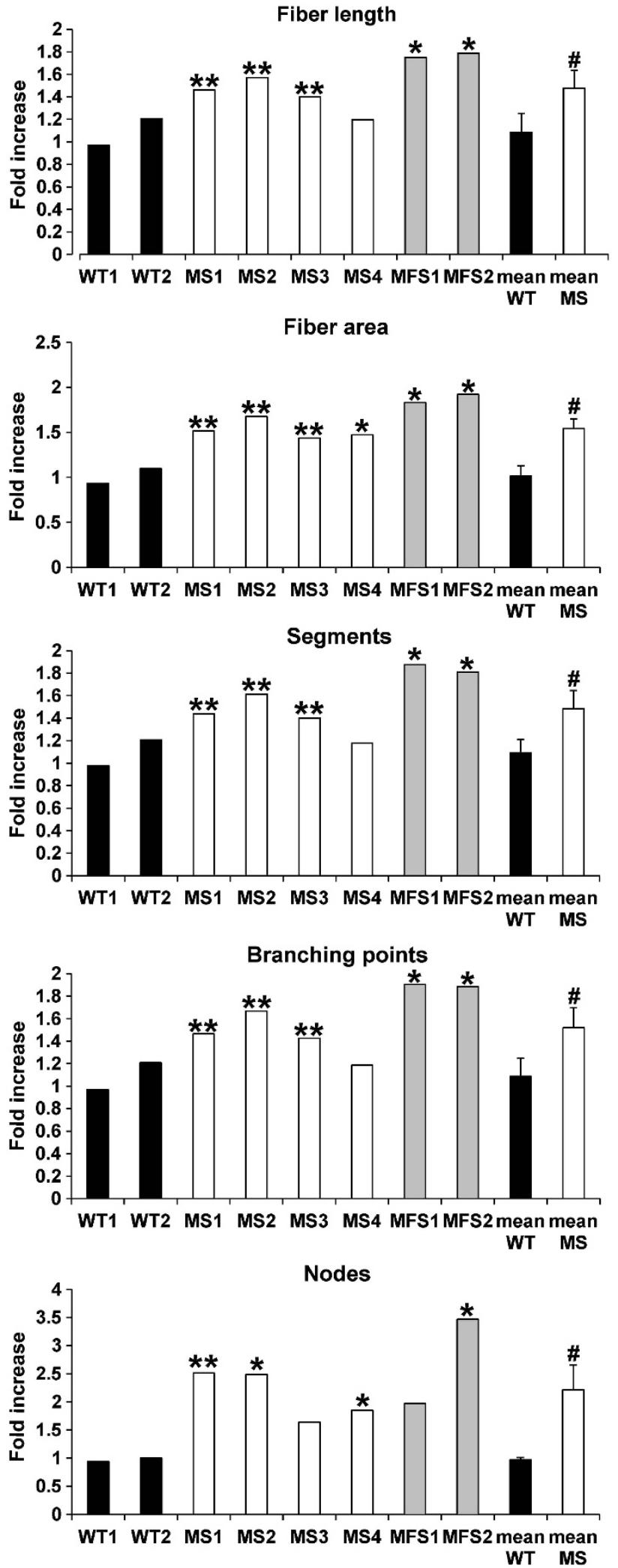

Figure 6 Quantification of microfibril network in losartan-treated vs untreated cells. MS fibroblasts treated with losartan showed a significant increase in fiber length, fiber area, number of segments, nodes, and branching points compared with vehicle (DMSO)-treated cells. Values are expressed as fold increase over vehicle-treated cells ( $t$-test: ${ }^{*} P<0.05$ and ${ }^{*} P<0.01$ vs corresponding DMSO-treated cells; ${ }^{\#} P<0.05$ vs mean WT).
(Figure 4). Taken together, these data suggest that losartan restores the balance of expression between proteases and inhibitors.

To investigate the consequences of losartan treatment on ECM deposition, we stained MS fibroblasts cultured for 14 days for FBN1 and COL1A1 in the presence of losartan or vehicle (DMSO). Skin fibroblasts from MFS patients and from healthy subjects (WT) were used as controls. Losartan-treated MS and MFS fibroblasts showed a clear improvement in microfibril deposition compared with vehicle-treated cells as shown by FBN1 staining, whereas no differences were observed in losartan-treated WT cells compared with DMSO-treated cells (Figure 5). No changes in COL1A1 staining in MS, MFS, and control cells were observed following incubation with losartan (Figure 5). To confirm ECM amelioration by losartan, we evaluated several parameters of the microfibril network, such as fiber length and area, branching points, segments, and nodes. Although no significant changes were detected in healthy control cells either treated with losartan or untreated (Figure 6), losartan-treated MS fibroblasts harboring the c.1498A $>\mathrm{G}$ (p.Ile500Val) mutation (MS1, MS2, and MS3) showed a significant $(t$-test: $P<0.01)$ increase in all tested parameters (Figure 6) compared with DMSO-treated cells, and MS4 displayed a significant $(t$-test: $P<0.05)$ increase only for the fiber area and the number of nodes (Figure 6). When grouped together losartan-treated MS fibroblasts improved in all microfibril network markers similar to MFS fibroblasts (Figure 6).

\section{DISCUSSION}

TGF- $\beta$ has a key role in the skeletal growth as demonstrated by the discovery of the molecular bases of several bone disorders affecting TGF- $\beta$ signaling. ${ }^{4}$ On the basis of the clinical overlap with other TGF$\beta$-related disease and the common perturbation of TGF- $\beta$ signaling, we hypothesized that MS fibroblasts will exhibit an ECM deposition defect. We first showed that four different MS primary fibroblasts bearing two different mutations [c.1486C $>\mathrm{T}$ (p.Arg496Cys) and c.1498A $>$ G (p.Ile500Val)] show increase in SMAD4 and phosphorylated SMAD2/total SMAD2 ratio and, consistent with previous studies, ${ }^{6}$ altered expression of both TGF- $\beta$ and BMP target genes. The two mutations exert different effects on TGF- $\beta$ and BMP target gene expression with c.1486C $>\mathrm{T}$ (p.Arg496Cys) mutation resulting only in MMP overexpression, whereas c.1498A $>\mathrm{G}$ (p.Ile500Val) mutation results in dysregulation of both MMPs and related inhibitors. Noteworthy, the Arg496 residue is directly involved in SMAD4 transcriptional activation. ${ }^{29}$ Next, we showed by FBN1 staining that MS fibroblasts have impaired microfibril deposition that appear to be less severe compared with MFS fibroblasts, whereas deposition of other ECM elements, such as type I collagen, is unaffected.

FBN1 is a key regulator of TGF- $\beta$ and BMP signaling because microfibrils form a scaffold that binds latent TGF- $\beta$ and BMP complexes and sequester them in an inactive state. ${ }^{30}$ Disruption of the microfibril network results in increased bioavailability of TGF- $\beta$ and BMP molecules, downstream activation of their signaling cascades, and feedback modulation of ECM that in turn aggravates matrix disorganization. ${ }^{31}$ This positive feedback loop mechanism has a major role in the pathogenesis of diseases caused by FBN1 mutations. ${ }^{31}$ However, FBN1 mutations also affect the structural integrity of FBN1 that likely has a role in disease pathogenesis. ${ }^{31}$ In contrast, ECM disorganization in MS due to SMAD4 mutations is only secondary to perturbation of the TGF- $\beta /$ SMAD pathway without intrinsic effects on the integrity of ECM proteins. This might explain the less severe microfibril defect of MS fibroblasts compared with MFS. The microfibril defect in MS 
fibroblasts is likely resulting from the unbalance between proteases and inhibitors, such as MMPs that are major determinants of ECM integrity. ${ }^{32}$ We found that treatment with losartan restored balanced expression of proteases and their inhibitors. Importantly, we observed a clear improvement of microfibril deposition in MS fibroblasts and demonstrated that losartan treatment significantly ameliorates microfibril network quality by improving fiber length, density, and networking. Whether this improvement will be reflected in amelioration of the disease phenotype and which abnormalities are amenable for correction by losartan remains to be studied. Nevertheless, the results of these results suggest a potential application of losartan for treatment of connective tissue manifestations of patients harboring SMAD4 mutations.

\section{CONFLICT OF INTEREST}

The authors declare no conflict of interest.

\section{ACKNOWLEDGEMENTS}

We thank the 'Cell Line and DNA Biobank from Patients Affected by Genetic Diseases' (G. Gaslini Institute) and the GDB bank (project GTF08022) of Telethon Genetic Biobank Network (Project NO. GTB07001) for providing fibroblasts from an affected patient and from wild-type controls. We thank Annamaria Carissimo and Luisa Cutillo from TIGEM Bioinformatic core for statistical and image analyses and Giorgio Modesti for assistance with MetaMorph software. This work was supported by the Fondazione Telethon, (TCBP37TELC and TCBMT3TELD to NB-P) and by the Italian Ministry of Health (GR-2009-1594913 to NB-P).

1 Myhre SA, Ruvalcaba RH, Graham CB: A new growth deficiency syndrome. Clin Genet $1981 ; 20: 1-5$

2 McGowan R, Gulati R, McHenry P et al: Clinical features and respiratory complications in Myhre syndrome. Eur J Med Genet 2011; 54: e553-e559.

3 Caputo V, Cianetti L, Niceta $\mathrm{M}$ et al: A restricted spectrum of mutations in the SMAD4 tumor-suppressor gene underlies Myhre syndrome. Am J Hum Genet 2012 90: 161-169.

4 Le Goff C, Cormier-Daire V: From tall to short: the role of TGFbeta signaling in growth and its disorders. Am J Med Genet C Semin Med Genet 2012; 160C: 145-153.

5 Loeys BL, Gerber EE, Riegert-Johnson D et al: Mutations in fibrillin-1 cause congenital scleroderma: stiff skin syndrome. Sci Transl Med 2010; 2: 23ra20.

6 Le Goff C, Mahaut C, Abhyankar A et al: Mutations at a single codon in Mad homology 2 domain of SMAD4 cause Myhre syndrome. Nat Genet 2012; 44: 85-88.

7 Lindor NM, Gunawardena SR, Thibodeau SN: Mutations of SMAD4 account for both LAPS and Myhre syndromes. Am J Med Genet A 2012; 158A: 1520-1521.

8 Asakura Y, Muroya K, Sato T, Kurosawa K, Nishimura G, Adachi M: First case of a Japanese girl with Myhre syndrome due to a heterozygous SMAD4 mutation. Am J Med Genet A 2012; 158A: 1982-1986.

9 Ross S, Hill CS: How the Smads regulate transcription. Int J Biochem Cell Biol 2008 40: 383-408.

10 Schutte M, Hruban RH, Hedrick L et al: DPC4 gene in various tumor types. Cancer Res 1996; 56: 2527-2530.
11 Gallione C, Aylsworth AS, Beis J et al: Overlapping spectra of SMAD4 mutations in juvenile polyposis (JP) and JP-HHT syndrome. Am J Med Genet A 2010; 152A 333-339.

12 Andrabi S, Bekheirnia MR, Robbins-Furman P, Lewis RA, Prior TW, Potocki L: SMAD4 mutation segregating in a family with juvenile polyposis, aortopathy, and mitral valve dysfunction. Am J Med Genet A 2011; 155A: 1165-1169.

13 Titomanlio L, Marzano MG, Rossi E et al: Case of Myhre syndrome with autism and peculiar skin histological findings. Am J Med Genet 2001; 103: 163-165.

14 Gonen M, Johnson WO, Lu Y, Westfall PH: The Bayesian two-sample t-test, Vol. Working paper 1, 2005.

15 Long X, Miano JM: Transforming growth factor-beta1 (TGF-beta1) utilizes distinct pathways for the transcriptional activation of microRNA 143/145 in human coronary artery smooth muscle cells. J Biol Chem 2011; 286: 30119-30129.

$16 \mathrm{He}$ W, Dorn DC, Erdjument-Bromage H, Tempst P, Moore MA, Massague J: Hematopoiesis controlled by distinct TIF1gamma and Smad4 branches of the TGFbeta pathway. Cell 2006; 125: 929-941.

17 Seong HA, Jung $\mathrm{H}$, Kim KT, Ha H: 3-Phosphoinositide-dependent PDK1 negatively regulates transforming growth factor-beta-induced signaling in a kinase-dependent manner through physical interaction with Smad proteins. J Biol Chem 2007; 282 12272-12289.

18 Choudhary C, Kumar C, Gnad F et al: Lysine acetylation targets protein complexes and co-regulates major cellular functions. Science 2009; 325: 834-840.

19 Moren A, Hellman U, Inada Y, Imamura T, Heldin $\mathrm{CH}$, Moustakas A: Differential ubiquitination defines the functional status of the tumor suppressor Smad4. J Biol Chem 2003; 278: 33571-33582.

20 Massam-Wu T, Chiu M, Choudhury R et al: Assembly of fibrillin microfibrils governs extracellular deposition of latent TGF beta. J Cell Sci 2010; 123: 3006-3018.

21 Lu P, Takai K, Weaver VM, Werb Z: Extracellular matrix degradation and remodeling in development and disease. Cold Spring Harb Perspect Biol 2011; 3: 1-24.

22 Ashworth JL, Murphy G, Rock MJ et al: Fibrillin degradation by matrix metalloproteinases: implications for connective tissue remodelling. Biochem J 1999; 340:Pt 1 $171-181$.

23 Yang J, Zheng J, Wu L et al: NDRG2 ameliorates hepatic fibrosis by inhibiting the TGF-beta1/Smad pathway and altering the MMP2/TIMP2 ratio in rats. PLoS One 2011; 6: e27710.

24 Wiercinska E, Naber HP, Pardali E, van der Pluijm G, van Dam H, ten Dijke P: The TGF-beta/Smad pathway induces breast cancer cell invasion through the up-regulation of matrix metalloproteinase 2 and 9 in a spheroid invasion model system. Breast Cancer Res Treat 2011; 128: 657-666.

25 Hyc A, Osiecka-Iwan A, Niderla-Bielinska J, Moskalewski S: Influence of LPS TNF, TGF-ss1 and IL-4 on the expression of MMPs, TIMPs and selected cytokines in rat synovial membranes incubated in vitro. Int J Mol Med 2011; 27 127-137.

26 Brooke BS, Habashi JP, Judge DP, Patel N, Loeys B, Dietz HC 3rd: Angiotensin II blockade and aortic-root dilation in Marfan's syndrome. N Engl J Med 2008; 358 2787-2795

27 Rodriguez-Vita J, Sanchez-Lopez E, Esteban V, Ruperez M, Egido J, Ruiz-Ortega M Angiotensin II activates the Smad pathway in vascular smooth muscle cells by a transforming growth factor-beta-independent mechanism. Circulation 2005; 111 2509-2517

28 Habashi JP, Doyle JJ, Holm TM et al: Angiotensin II type 2 receptor signaling attenuates aortic aneurysm in mice through ERK antagonism. Science 2011; 332: 361-365.

29 Chacko BM, Qin B, Correia JJ, Lam SS, de Caestecker MP, Lin K: The L3 loop and C-terminal phosphorylation jointly define Smad protein trimerization. Nat Struc Biol 2001; 8: 248-253.

30 Ramirez F, Rifkin DB: Extracellular microfibrils: contextual platforms for TGFbeta and BMP signaling. Curr Opin Cell Biol 2009; 21: 616-622.

31 Doyle JJ, Gerber EE, Dietz HC: Matrix-dependent perturbation of TGFbeta signaling and disease. FEBS Lett 2012; 586: 2003-2015.

32 Aiken A, Khokha R: Unraveling metalloproteinase function in skeletal biology and disease using genetically altered mice. Biochim Biophys Acta 2010; 1803 $121-132$.

Supplementary Information accompanies this paper on European Journal of Human Genetics website (http://www.nature.com/ejhg) 